\title{
Gender Differences in Mental Health Outcomes Amid the COVID-19 Pandemic and a Collapsing Economy: A Cross-Sectional Study
}

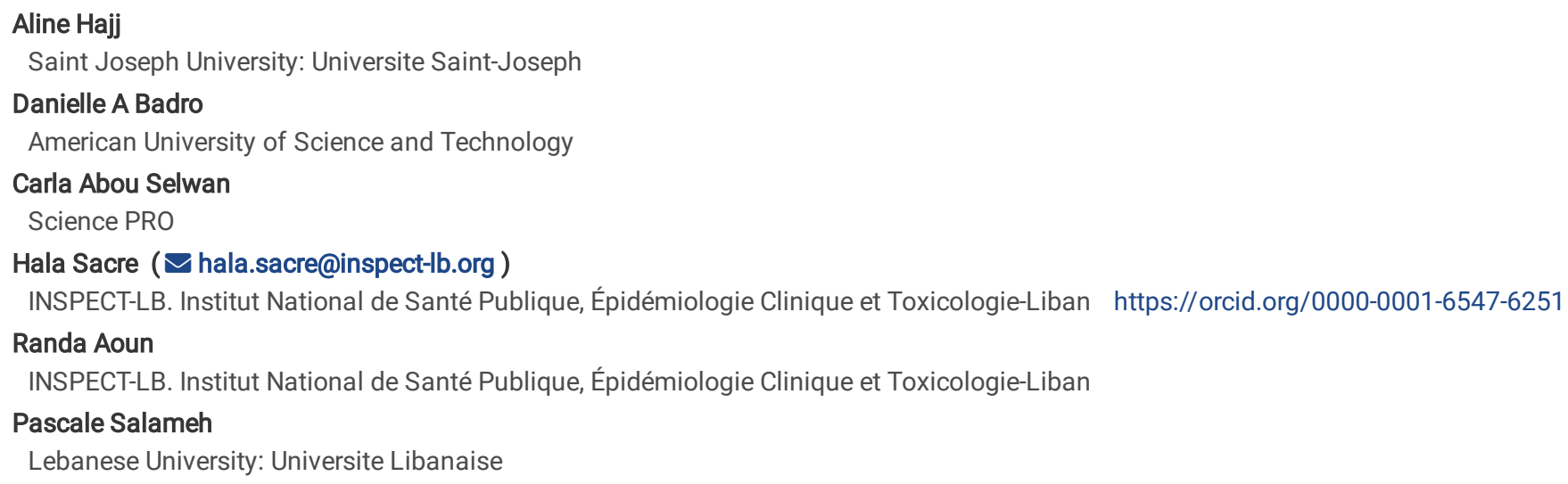




\section{Abstract}

Background: Emergency plans to contain the COVID-19 failed to take into consideration the repercussions of the combined economic and sanitary crisis among the most vulnerable populations who might be disproportionately affected, including women and abused people. This study primarily aimed to assess the effects of the COVID-19 and economy-related variables on Lebanese adults' mental health, focusing on gender differences.

Methods: An online-based cross-sectional study was conducted between May 10-20, 2020, using validated scales. All adults living in Lebanon, with access to the internet, were eligible, regardless of their age, socioeconomic status, race, ethnicity, nationality, sex, gender identity, religion, geography, ability, and culture $(\mathrm{n}=502)$.

Results: The multivariate analysis, adjusted on sociodemographic characteristics, economic, and coronavirus-related variables, showed that women exhibit higher levels of stress, anxiety, and post-traumatic stress symptoms (PTSS) than men. Moreover, violence at home was associated with more stress and insomnia, with borderline results for anxiety and well-being. No significant difference was found for PTSS.

Conclusion: In the context of combined health and economic crises, mental health outcomes are not equally felt by women. Additional studies in a few months would help better understand the long-term impact, especially on PTSS and QOL, which might not be perceptible during the crisis. Focused public health strategies are warranted to support and protect the most vulnerable populations.

\section{Introduction}

Since the first reported COVID-19 positive case in Lebanon on February 21, 2020, the government and the Ministerial Committee of Coronavirus Prevention implemented an emergency plan to contain the spread of the disease, leading to the sanitary lockdown on March 15, 2020 [1]. These measures ranked Lebanon among the countries that succeeded, at first, in handling the "immediate health-care problems" related to COVID-19 [2]. However, some gender experts in Lebanon considered that the action plan failed to take into consideration the repercussions of the combined economic and sanitary crisis among the most vulnerable populations, including women and abused people who might be disproportionately affected $[3,4]$.

Indeed, women and men might not equally experience the negative consequences of long confinement duration, fear of infection, frustration, boredom, inadequate supplies, inadequate information, financial loss, and stigma. In Lebanon, in particular, it is estimated that $58 \%$ of working women have informal, unregulated, or unprotected by labor laws job, which makes them more vulnerable to both infection and poverty, with the looming threat of unemployment [4]. Moreover, the current pandemic situation, with the confinement of families at home, has led to an increased workload in the domestic chores women are expected to carry out, which could consume them physically. Finally, since the closure of schools on February 28,2020 [5], women had to carry their children homeschooling and ensure a "state of tranquility", whether for children learning or men online working. As a result, women might not be able to engage and deliver their "formal job", which may limit their economic opportunities [6].

Domestic violence is another major issue that may have stark downstream outcomes on the mental, and physical health of women, especially when forced into confinement with a potentially violent partner [7-11]. Studies have shown that reported domestic violence often substantially increased after a catastrophic event [12]. During this pandemic, several reports shed light on a global increase in domestic violence and women abuse [13-15]. In Lebanon, few studies have been previously published exploring the prevalence of violence against women [16-18]. Authors identified, among large sample sizes, rates up to $35 \%$ of overall domestic violence (in 2002) [17], 37.1\% of physical violence and $49.4 \%$ of non-physical violence (in 2020) [16].

Soon since the beginning of the COVID-19, Abaad, a not-for-profit organization, reported that home violence complaints had doubled over the first months of 2020 compared to one year prior (reaching 500 versus 270 in the same period of 2019) [4]. Several factors could be accounted for this increased violence, including confinement and movement restrictions, but also isolation, frustration, overcrowding, and psychological factors such as stress and anxiety [7, 9-11, 19]. The World Health Organization (WHO) published a statement calling for action to protect and support vulnerable persons overcome such "hidden crises of the COVID-19 pandemic". These recommendations should be further reinforced in a country such as Lebanon where growing economic vulnerability is thought to increase the threat of a potential harm $[9,19]$.

Thus, women are expected to have lower mental health and overall well-being. Indeed, recent studies highlighted that the female gender was significantly associated with a higher risk of developing coronavirus-related sleep disorders [20], distress [20], anxiety [21], post-traumatic stress symptoms [22]. However, none has evaluated the impact of gender differences, domestic violence, and economic hardship on several sociodemographic-adjusted health outcomes, namely, sleep, anxiety, distress, post-traumatic stress disorder (PTSD), and well-being. Therefore, this study aimed at assessing the effects of the COVID-19 and economic-related variables on Lebanese adults' mental health, focusing on gender differences; a secondary objective is to assess the role of domestic violence in that context.

\section{Methods}

\section{Study design and sampling.}

This cross-sectional study was conducted between May 10 and 20, 2020, using an online-based questionnaire created on Google forms. Due to the government-mandated sanitary lockdown, the snowball sampling technique was used to distribute the survey to participants through social media 
platforms and WhatsApp groups. All adults living in Lebanon, with access to the internet, were eligible, regardless of their age, socioeconomic status, race, ethnicity, nationality, sex, religion, geography, ability, and culture. A total of 502 respondents filled out the questionnaire that required between 15 and 20 minutes to complete.

\section{Minimal sample size calculation.}

The minimum sample size was calculated using the G-Power software, version 3.0.10.

The calculated effect size was 0.0526 , expecting a squared multiple correlation of $0.05\left(R^{2}\right.$ deviation from 0$)$ related to the Omnibus test of multiple regression. The minimum necessary sample was $n=454$, considering an alpha error of $5 \%$, a power of $80 \%$, and allowing 25 predictors to be included in the model.

\section{Questionnaire.}

The online questionnaire was available in Arabic, the native language in Lebanon. It consisted of three parts. The first part assessed the sociodemographic features of the participants, such as age, gender, marital status, educational level, employment status, region, current household monthly income, divided into five levels, according to the official exchange rate ( 1 USD $=1500$ LBP): no income, low < 675,000LBP (450 USD, the official lowest wage in Lebanon), moderate 675,000-1,500,000LBP (450-1,000 USD), high 1,500,000-3,000,000 LBP (1,000-2,000 USD), and very high income > $3,000,000$ LBP (2,000 USD). It also included questions about medical coverage, smoking, alcohol consumption, self-perception of the financial situation, having been infected or in contact with people contaminated with coronavirus, and physical activity before and during the COVID-19 pandemic. Violence was self-evaluated by the answer to the following questions with four possible answers: 1-Yes; 2-No; 3-No answer; 4-Does not apply:

Is there any form of verbal violence in your home? Is there any form of physical violence in your home? Is there any form of sexual violence in your home? Is there any form of any other type of violence in your home?".

The second part of the questionnaire consisted of a set of 20 work-related questions, addressed to working people and those seeking employment and aiming to explore to what extent their current employment was affected by either the economic crisis or the COVID-19 pandemic. Examples of the questions asked: Do you have to go out to make a living despite the sanitary lockdown? Are you able to apply social distancing while working (1.5-2m safety distance)? Did your company change the working hours because of the economic crisis or the COVID-19 pandemic? Has your salary/income been affected by the economic crisis or the COVID-19 pandemic? Are you worried about the long-term impact of the economic crisis or the COVID-19 pandemic on your business/job? Did the economic crisis or the COVID-19 pandemic result in decreasing the salaries of employees? Did the economic crisis or the COVID-19 pandemic cause the dismissal of some employees? What were the criteria used to lay-off employees?

Finally, the third part of the questionnaire included several validated scales:

The fear of COVID-19 scale.

This 7-item tool measures the extent of fear of the COVID-19 in adults [23]. The total score, ranging from 1 to 35 , is calculated by summing the answers to all questions graded from 1 (strongly disagree) to 5 (strongly agree) on a 5-point Likert scale. Higher scores indicate a greater fear of COVID-19 $\left(a_{\text {Cronbach }}=0.893\right)$.

The Beirut Distress Scale-22 (BDS-22).

The BDS-22, validated in Lebanon [24], evaluates the level of stress in the general Lebanese adult population. It consists of 22 questions exploring six different domains: depressive symptoms, demotivation, psychosomatic symptoms, mood deterioration, intellectual inhibition, and anxiety. Responses are rated on a 4-point Likert scale from 0 (not at all) to 3 (all of the times), with higher scores indicating higher levels of stress. $\left(a_{\text {Cronbach }}=0.965\right)$.

The Lebanese Anxiety Scale-10 (LAS-10).

The LAS-10 is a 10-item scale validated in Lebanon [25] used to screen for anxiety in the general Lebanese adult population. Questions 1 to 7 are scored on a 5-point Likert scale from 0 (not present) to 4 (very severe), whiles items 8-10 are graded on a 4-point Likert scale from 1 (never/almost never) to 4 (almost always). Higher scores indicate higher anxiety. The Cronbach's alpha for this scale was 0.919.

The of Posttraumatic Stress Disorder Checklist for DSM-5 (PCL-5).

This 20-item tool evaluates the 20 DSM-5 symptoms of PTSD in the past month. It is available in Arabic language as was validated in Syria [26]. Responses are rated on a 5-point Likert scale from 0 (not at all) to 4 (Extremely). The total symptom severity score (range $0-80$ ) is obtained by summing the responses for each of the 20 items. Higher scores reflect higher symptoms resulting from a stressful experience $\left(a_{C r o n b a c h}=0.971\right)$. In this manuscript, "PTSS" is used for both the symptoms and the disorder, as the questionnaire evaluated the symptoms more likely than the disorder.

The Lebanese Insomnia Scale (LIS-18). 
This 18-item tool, recently validated in Lebanon [27], is used to screen for insomnia. Answers are graded on a 5-point Likert scale from 1 (Never) to 5 (Always), with higher scores indicating higher insomnia. $\left(\mathrm{a}_{\text {Cronbach }}=0.847\right)$.

The Family APGAR Index.

This instrument evaluates satisfaction with the global family function [28]. It consists of five questions graded on a 3-point Likert scale: 0 (hardly ever), 1 (some of the time), and 2 (almost always). Each corresponds to a component of family function, i.e., Adaptation, Partnership, Growth, Affection, and Resolve (APGAR). The total, ranging from 0 to 10, is obtained by summing the answers to all items. Higher scores indicate higher satisfaction with family function $\left(\mathrm{a}_{\text {Cronbach }}=0.927\right)$.

The World Health Organization-Five Well-Being Index (WHO-5).

This short version, validated in Lebanon [29], consists of five questions graded from 0 to 5 assessing mental well-being in the past month. The total score ranges from 0 to 25 ; higher scores indicate a better mental well-being $\left(a_{\text {cronbach }}=0.796\right)$.

The InCharge Financial Distress/Financial Well-Being Scale (IFDFW).

This tool includes eight items assessing the perceived financial distress/financial well-being on a 1-to-10 linear scale [30]. Lower scores reflect higher financial distress and lower well-being $\left(a_{\text {cronbach }}=0.925\right)$. Since this tool is copyrighted, written permission was obtained from the authors to use it and validate it in Lebanon.

Translation Procedure and Piloting.

Three of the scales (fear of the COVID-19, the Family APGAR Index, and the IFDFW), not validated and available in Arabic, were translated into this language. Three authors performed the forward translation, and the other three, the back translation. Discrepancies between original English versions and translated ones were resolved by consensus. The final version was pilot-tested on ten people unfamiliar with the study. The final dataset did not include their answers.

\section{Statistical Analysis.}

Data were collected using Google Forms, a tool that automatically generates an Excel database, then transferred to IBM SPSS ${ }^{\circ}$, version 23.0 for analysis. Prior to the analysis, data were weighted according to gender, age, and dwelling region, based on the figures of the Central Administration of Statistics [31]. In the descriptive analysis, frequencies and percentages were used for categorical variables, and means and standard deviations for quantitative variables. For dependent variables (BDS-22, LIS-18, LAS-10, PCL-5, and WHO-5), the distribution was considered normal based on the visual inspection of the histogram, and the skewness and kurtosis (lower than 1). These conditions are compatible with normality in a sample size higher than 300 [32].

For the bivariate analysis of continuous variables, the Student's T-test was used to compare the means between two groups, after checking for homogeneity of variances using the Levene's test. In case the variances were not homogeneous, the corrected T-Test was applied. The Chi-square test was used for dichotomous and multinomial variables. When calculated values per case were $<5$, the Fisher exact test was performed. In all cases, a pvalue lower than 0.05 was considered significant.

Afterward, a multivariate analysis employing the General Linear Model was performed, using the mental health-related dependent variables; the ENTER method was applied to come up with the appropriate model with proper assumptions. This method allowed to calculate the estimated marginal means for mental health measures among men and women, and among subjects with and without declared domestic violence.

\section{Results}

\section{Sociodemographic characteristics and gender distribution.}

There were slightly more females (52.68\%) than males in the sample. Males were significantly more educated $(92 \%$ vs. $85 \%$ university degree; $p=$ 0.018), with higher percentages of previous, occasional, and regular cigarette and waterpipe smokers. Males also reported more domestic violence (8.4\% vs. 3.8\%; $p=0.038)$. As expected, significantly more women $(17 \% ; p<0.001)$ have never been employed, versus $2.9 \%$ for men (Table 1$)$. 
Table 1

Sociodemographic characteristics and gender distribution

\begin{tabular}{|c|c|c|c|}
\hline Characteristic & $\begin{array}{l}\text { Males } \\
\mathrm{N}=238(100 \%)\end{array}$ & $\begin{array}{l}\text { Females } \\
\mathrm{N}=265(100 \%)\end{array}$ & p-value \\
\hline Marital status & $92(38.8 \%)$ & $97(36.6 \%)$ & 0.114 \\
\hline Single & $139(58.6 \%)$ & $151(57.0 \%)$ & \\
\hline Married & $6(2.5 \%)$ & $17(6.4 \%)$ & \\
\hline \multicolumn{4}{|l|}{ Widowed/Divorced } \\
\hline Level of education & $19(8.0 \%)$ & $39(14.7 \%)$ & 0.018 \\
\hline Less than university & $219(92.0 \%)$ & $226(85.3 \%)$ & \\
\hline \multicolumn{4}{|l|}{ University degree } \\
\hline Dwelling region & $39(16.4 \%)$ & $45(17.0 \%)$ & 0.671 \\
\hline Beirut (capital) & $110(46.2 \%)$ & $112(42.3 \%)$ & \\
\hline Mount Lebanon & $35(14.7 \%)$ & $34(12.8 \%)$ & \\
\hline South Lebanon & $19(8.0 \%)$ & $29(10.9 \%)$ & \\
\hline Bekaa plain & $35(14.7 \%)$ & $45(17.0 \%)$ & \\
\hline \multicolumn{4}{|l|}{ North Lebanon } \\
\hline Household size & $77(32.5 \%)$ & $84(31.8 \%)$ & 0.973 \\
\hline Lower than 4 persons & $66(27.8 \%)$ & $70(26.5 \%)$ & \\
\hline 4 persons & $56(23.6 \%)$ & $66(25.0 \%)$ & \\
\hline 5 persons & $38(16.0 \%)$ & $44(16.7 \%)$ & \\
\hline \multicolumn{4}{|l|}{6 and more } \\
\hline Number of dependent children & $101(42.6 \%)$ & $105(39.8 \%)$ & 0.357 \\
\hline None & $16(6.8 \%)$ & $29(11.0 \%)$ & \\
\hline 1 child & $66(27.8 \%)$ & $66(25.0 \%)$ & \\
\hline 2 children & $54(22.8 \%)$ & $64(24.2 \%)$ & \\
\hline \multicolumn{4}{|l|}{3 or more } \\
\hline Number of rooms & $79(33.2 \%)$ & $88(33.3 \%)$ & 0.611 \\
\hline$<5$ rooms & $70(29.4 \%)$ & $69(26.1 \%)$ & \\
\hline 5 rooms & $53(22.3 \%)$ & $56(21.2 \%)$ & \\
\hline 6 rooms & $36(15.1 \%)$ & $51(19.3 \%)$ & \\
\hline \multicolumn{4}{|l|}{7 or more } \\
\hline Alcohol consumption & $11(4.6 \%)$ & $3(1.1 \%)$ & $<0.001$ \\
\hline Previous & $70(29.4 \%)$ & $127(48.1 \%)$ & 0.001 \\
\hline None & $30(12.6 \%)$ & $14(5.3 \%)$ & Ref \\
\hline Occasional & $127(53.4 \%)$ & $120(45.5 \%)$ & 0.001 \\
\hline Regular & & & $<0.001$ \\
\hline Cigarette smoking & $11(4.6 \%)$ & $10(3.8 \%)$ & 0.059 \\
\hline Previous & $145(61.2 \%)$ & 189(71.1\%) & \\
\hline None & $37(15.6 \%)$ & $23(8.6 \%)$ & \\
\hline Occasional & $44(18.6 \%)$ & $44(16.5 \%)$ & \\
\hline Regular & & & \\
\hline
\end{tabular}




\begin{tabular}{|llll|}
\hline Characteristic & Males & Females & p-value \\
& N=238(100\%) & N=265(100\%) & \\
\hline Waterpipe smoking & $22(9.3 \%)$ & $5(1.9 \%)$ & $<0.001$ \\
Previous & $153(64.6 \%)$ & $210(79.2 \%)$ & $<0.001$ \\
None & $22(9.3 \%)$ & $11(4.2 \%)$ & Ref \\
Occasional & $40(16.9 \%)$ & $39(14.7 \%)$ & 0.168 \\
Regular & & & 0.007 \\
\hline Self-reported domestic violence & $215(90.7 \%)$ & $247(93.6 \%)$ & 0.031 \\
No violence & $20(8.4 \%)$ & $10(3.8 \%)$ & Ref \\
Yes violence & $2(0.8 \%)$ & $7(2.7 \%)$ & 0.038 \\
No answer & & & 0.188 \\
\hline Professional status & $184(77.3 \%)$ & $161(60.8 \%)$ & $<0.001$ \\
Works currently & $7(2.9 \%)$ & $45(17.0 \%)$ & Ref \\
Housewife/never works & $21(8.8 \%)$ & $29(10.9 \%)$ & $<0.001$ \\
Student & $17(7.1 \%)$ & $23(8.7 \%)$ & 0.134 \\
Retired & $9(3.8 \%)$ & $7(2.6 \%)$ & 0.194 \\
Looking for a job & & & 0.819 \\
\hline Age in years & Mean (SD) & Mean(SD) & 0.717 \\
\hline
\end{tabular}

\section{Economic characteristics and gender distribution.}

The subjective assessment before the COVID-19 did not significantly differ between males and females; however, regarding the post-COVID-19 crisis evaluation, more males classified themselves in poorer classes, although more males belonged to households with higher revenues. Overall, socioeconomic quartiles were equally distributed between males and females. There were significantly more males with no health coverage (Table 2). 
Table 2

Economic characteristics and gender distribution

\begin{tabular}{|c|c|c|c|}
\hline Characteristic & $\begin{array}{l}\text { Males } \\
\mathrm{N}=238(100 \%)\end{array}$ & $\begin{array}{l}\text { Females } \\
\mathrm{N}=265(100 \%)\end{array}$ & p-value \\
\hline Subjective assessment before COVID & 0 & $5(1.9 \%)$ & 0.124 \\
\hline No answer & $14(5.9 \%)$ & $17(6.4 \%)$ & \\
\hline Rich & $213(89.9 \%)$ & $234(88.6 \%)$ & \\
\hline Middle class & $4(1.7 \%)$ & $6(2.3 \%)$ & \\
\hline Middle to low & $6(2.5 \%)$ & $2(0.8 \%)$ & \\
\hline \multicolumn{4}{|l|}{ Below poverty line } \\
\hline Subjective assessment after COVID & $4(1.7 \%)$ & $9(3.4 \%)$ & 0.018 \\
\hline No answer & 0 & $5(1.9 \%)$ & 0.016 \\
\hline Rich & $151(63.7 \%)$ & $176(66.7 \%)$ & 0.006 \\
\hline Middle class & $68(28.7 \%)$ & $69(26.1 \%)$ & 0.020 \\
\hline Middle to low & $14(5.9 \%)$ & $5(1.9 \%)$ & 0.049 \\
\hline Below poverty line & & & Ref \\
\hline Current Health Coverage & $32(13.5 \%)$ & $20(7.6 \%)$ & $<0.001$ \\
\hline No Health coverage & $69(29.1 \%)$ & $86(32.6 \%)$ & Ref \\
\hline Private insurance & $108(45.6 \%)$ & $97(36.7 \%)$ & 0.252 \\
\hline Social security & $28(11.8 \%)$ & $61(23.1 \%)$ & 0.034 \\
\hline Other public coverage & & & $<0.001$ \\
\hline Household income & $3(1.3 \%)$ & $12(4.5 \%)$ & 0.029 \\
\hline Less than $675,000 \mathrm{LP}$ & $23(9.7 \%)$ & $41(15.5 \%)$ & Ref \\
\hline $675,000-1,500,000 \mathrm{LP}$ & $76(32.1 \%)$ & $73(27.5 \%)$ & 0.362 \\
\hline $1,500,000-3,000,000 \mathrm{LP}$ & $135(57.0 \%)$ & $139(52.5 \%)$ & 0.022 \\
\hline More than $3,000,000 \mathrm{LP}$ & & & 0.027 \\
\hline Socioeconomic quartile & $55(23.3 \%)$ & $78(30.1 \%)$ & 0.356 \\
\hline Quartile 1 & $70(29.7 \%)$ & $72(27.8 \%)$ & \\
\hline Quartile 2 & $62(26.3 \%)$ & $57(22.0 \%)$ & \\
\hline Quartile 3 & $49(20.8 \%)$ & $52(20.1 \%)$ & \\
\hline Quartile 4 & & & \\
\hline
\end{tabular}

COVID-19 exposure, health characteristics, and gender distribution.

More males were in contact with COVID-19 cases (6.7\% vs. $0.8 \%$ ), knew someone infected with coronavirus (36.3\% vs. $22.3 \%)$, and were visiting/receiving friends and relatives during the lockdown; also, more males were doing physical activity (69\% vs. 59\%), and more had a chronic disease ( $25 \%$ vs. $17 \%)$. More females were afraid to go out to get treatment (19\% vs. $11 \%)$, and more were worried about a family member contracting the disease (Table 3). 
Table 3

COVID-19 exposure, health characteristics and gender distribution.

\begin{tabular}{|c|c|c|c|}
\hline Characteristic & $\begin{array}{l}\text { Males } \\
\mathrm{N}=\mathbf{2 3 8}(100 \%)\end{array}$ & $\begin{array}{l}\text { Females } \\
\mathrm{N}=265(100 \%)\end{array}$ & p-value \\
\hline No COVID-19 infection & 236(99.2\%) & 263(99.6\%) & 0.606 \\
\hline Had COVID-19 infection & $2(0.8 \%)$ & $1(0.4 \%)$ & \\
\hline Contact with COVID-19 (work, family, store) & $16(6.7 \%)$ & $2(0.8 \%)$ & $<0.001$ \\
\hline No contact with COVID-19 & $222(93.3 \%)$ & $263(99.2 \%)$ & \\
\hline Knows someone infected & $86(36.3 \%)$ & $59(22.3 \%)$ & $<0.001$ \\
\hline Does not know anybody infected & $151(63.7 \%)$ & $206(77.7 \%)$ & \\
\hline Visiting/receiving friends & $71(30.0 \%)$ & $38(14.3 \%)$ & $<0.001$ \\
\hline No visiting/receiving friends & $166(70.0 \%)$ & $227(85.7 \%)$ & \\
\hline Visiting/receiving family & $160(67.5 \%)$ & $151(57.0 \%)$ & 0.015 \\
\hline No visiting/receiving family & $77(32.5 \%)$ & $114(43.0 \%)$ & \\
\hline Physical activity & $165(69.3 \%)$ & $157(59.2 \%)$ & 0.019 \\
\hline No physical activity & $73(30.7 \%)$ & $108(40.8 \%)$ & \\
\hline Chronic disease & $58(24.5 \%)$ & $45(17.0 \%)$ & 0.038 \\
\hline No chronic disease & $179(75.5 \%)$ & $220(83.0 \%)$ & \\
\hline Regular treatment for chronic disease & $66(27.8 \%)$ & $61(23.0 \%)$ & 0.004 \\
\hline Yes & $28(11.8 \%)$ & $13(4.9 \%)$ & 0.067 \\
\hline No regular treatment & $143(60.3 \%)$ & $191(72.1 \%)$ & Ref \\
\hline Does not apply & & & 0.002 \\
\hline Fear no access to treatment & $74(31.1 \%)$ & $80(30.2 \%)$ & 0.651 \\
\hline No & $68(28.6 \%)$ & $68(25.7 \%)$ & \\
\hline Yes & $96(40.3 \%)$ & $117(44.2 \%)$ & \\
\hline \multicolumn{4}{|l|}{ Does not apply } \\
\hline Fear to go out get treatment & $124(52.3 \%)$ & $93(35.2 \%)$ & $<0.001$ \\
\hline No & $26(11.0 \%)$ & $51(19.3 \%)$ & Ref \\
\hline Yes & $87(36.7 \%)$ & $120(45.5 \%)$ & $<0.001$ \\
\hline Does not apply & & & 0.002 \\
\hline Family member has chronic disease & $91(38.2 \%)$ & $108(40.9 \%)$ & 0.566 \\
\hline No & $124(52.1 \%)$ & 137(51.9\%) & \\
\hline Yes & $23(9.7 \%)$ & $19(7.2 \%)$ & \\
\hline \multicolumn{4}{|l|}{ Does not apply } \\
\hline Worried family member gets COVID & $62(26.1 \%)$ & $34(12.9 \%)$ & 0.001 \\
\hline No & $118(49.6 \%)$ & $150(56.8 \%)$ & Ref \\
\hline Yes & $58(24.4 \%)$ & $80(30.3 \%)$ & 0.001 \\
\hline Does not apply & & & 0.001 \\
\hline
\end{tabular}

\section{Gender effects on self-declared measures}

In the bivariate analysis, females had significantly higher fear of COVID-19, fear of poverty, stress, anxiety, and PTSS, lower well-being, but similar insomnia, financial wellness, and family satisfaction scores (Table 4). Based on the multivariate analysis, the adjusted estimated marginal means showed lower means for stress, anxiety, and PTSS in men after adjustment on sociodemographic characteristics, economic, and coronavirus-related variables. However, the mental well-being was not significantly different between males and females (Fig. 1a). 
Table 4

Gender and violence effects on self-declared measures (Bivariate analysis)

\begin{tabular}{|c|c|c|c|c|c|c|c|c|c|}
\hline & $\begin{array}{l}\text { Fear of } \\
\text { Poverty }\end{array}$ & $\begin{array}{l}\text { Fear of } \\
\text { COVID-19 }\end{array}$ & $\begin{array}{l}\text { BDS-22 } \\
\text { (Stress) }\end{array}$ & $\begin{array}{l}\text { LAS-10 } \\
\text { (Anxiety) }\end{array}$ & $\begin{array}{l}\text { LIS-18 } \\
\text { (Insomnia) }\end{array}$ & $\begin{array}{l}\text { PCL-5 } \\
\text { (PTSS) }\end{array}$ & $\begin{array}{l}\text { WHO-5 } \\
\text { (Mental } \\
\text { well-being) }\end{array}$ & $\begin{array}{l}\text { IFDFW } \\
\text { Financial }\end{array}$ & $\begin{array}{l}\text { APGAR } \\
\text { family } \\
\text { scale }\end{array}$ \\
\hline Males & $6.53(2.75)$ & $10.58(6.06)$ & $13.42(14.45)$ & $14.40(9.29)$ & $43.77(11.60)$ & 13.57(14.77) & 15.61(5.03) & 41.38(16.81) & 7.76(2.73) \\
\hline Females & $7.23(2.52)$ & $12.03(5.92)$ & $18.49(15.83)$ & $16.10(8.32)$ & $45.36(10.87)$ & $21.29(18.04)$ & $14.08(4.74)$ & $38.58(17.71)$ & $7.85(2.72)$ \\
\hline p-value & 0.003 & 0.007 & $<0.001$ & 0.032 & 0.114 & $<0.001$ & $<0.001$ & 0.071 & 0.726 \\
\hline \multirow{2}{*}{$\begin{array}{l}\text { No } \\
\text { violence } \\
\text { (0) }\end{array}$} & $6.91(2.64)$ & $11.49(6.05)$ & 15.52(15.27) & 14.92(8.69) & $43.96(10.91)$ & 17.87(16.97) & $15.06(4.90)$ & 40.01(17.18) & $7.93(2.60)$ \\
\hline & $6.52(2.97)$ & $8.67(5.64)$ & $23.00(16.12)$ & $20.57(9.47)$ & $53.89(12.84)$ & $15.07(17.65)$ & $11.37(4.52)$ & $40.85(19.73)$ & $6.44(3.75)$ \\
\hline \multirow{2}{*}{$\begin{array}{l}\text { Any } \\
\text { violence } \\
\text { (1) }\end{array}$} & $7.64(2.01)$ & 12.74(3.91) & $22.12(13.61)$ & 16.82(8.73) & 47.07(9.38) & 14.51(17.30) & 13.17(3.51) & $32.08(16.17)$ & $6.13(3.32)$ \\
\hline & 0.502 & 0.036 & 0.017 & 0.003 & $<0.001$ & 0.577 & $<0.001$ & 0.347 & 0.069 \\
\hline \multirow{2}{*}{$\begin{array}{l}\text { No } \\
\text { answer } \\
(2)\end{array}$} & 1.000 & 0.040 & 0.030 & 0.002 & $<0.001$ & 1.000 & $<0.001$ & 1.000 & 0.150 \\
\hline & 1.000 & 1.000 & 0.540 & 0.732 & 1.000 & 1.000 & 0.678 & 0.465 & 0.061 \\
\hline \multicolumn{10}{|l|}{$\begin{array}{l}\text { ANOVA } \\
\text { p-value }\end{array}$} \\
\hline \multicolumn{10}{|l|}{$\begin{array}{l}\text { p-value } \\
\text { (1) vs } \\
\text { (0) }\end{array}$} \\
\hline $\begin{array}{l}\text { p-value } \\
\text { (2) vs } \\
\text { (0) }\end{array}$ & & & & & & & & & \\
\hline
\end{tabular}

\section{Violence effects on self-declared measures}

Individuals with domestic violence at home had a lower fear of COVID-19, lower mental well-being, and higher stress, anxiety, and insomnia; there was no significant difference for fear of poverty, financial wellness, and PTSS (Table 4). In the multivariate analysis, those with violence at home had higher stress and insomnia, with borderline results for anxiety and well-being, and no significant difference for PTSS (Fig. 1b).

\section{Discussion}

A gendered understanding of COVID-19 is highly valuable in the context of combined sanitary and economic crises, as it sheds light on the multiple and inter-related levels of inequality that shape vulnerability to infection and health-related outcomes.

Our study has shown that women reported significantly higher levels of stress, anxiety, and PTSS than men after adjustment over sociodemographic, economic, and coronavirus-related factors, suggesting that the impact of the current situation is worse on women. These results are not surprising since previous findings have revealed that anxiety disorders and depressive disorders are more frequent in women [33]. Gender-differences in psychiatric disorders were also reported during the actual COVID-19 pandemic. Indeed, studies showed that anxiety disorder was two to three-fold of higher levels in women than in men during the COVID-19 [20, 21, 34] [35]. As for PTSD, some authors have identified that being a female was the most potent predictor of PTSD symptoms after pandemics. In the sub-symptom analysis of the same PCL-5 used in our study, women had more reexperiencing, negative alterations in cognition or mood, and hyperarousal than men [22]. This outcome could be related to the higher reactivity of trauma-associated neural networks in women's brains, when exhibiting higher levels of stress, anxiety, and helplessness compared to men [36]. However, unlike an Italian study [20], women in our sample did not report increased COVID-19-related sleep disorders, nor deterioration in the overall well-being. Possible explanations are that the scales used to evaluate insomnia were different, or men and women share common triggering factors, diluted when performing the statistical adjustments in the multivariable analysis.

As for our secondary objective, to the best of our knowledge, no previous studies have assessed the differences in health outcomes based on the different types of violence during the COVID-19 pandemic. The recorded numbers of any violence type (verbal, physical, sexual, or other) are quite surprising since men reported domestic brutality more than women (8.4\% versus $3.8 \%$ ), at a time where additional $2.7 \%$ of women chose the option "no answer" for reporting violence, despite the survey being anonymous. This outcome outlines a hidden cultural stigma preventing women from disclosing abuse [37].

Although the rates reported in our study is lower than what was previously described in Lebanon [16, 17], probably due to the university level of education of the majority of our participants, we have been able to identify that this domestic violence was associated with higher levels of stress and insomnia, with borderline results for anxiety and mental well-being. The overall relationship between interpersonal violence and these factors has been described in the literature, even if not in the context of the COVID-19 pandemic [38, 39]. This outcome is expected in time of crisis and lockdown, and is a direct consequence of forced confinement with a violent partner, with decreased resources, enhanced psychological stress, increased negative coping mechanisms (such as alcohol or smoking), and reduced ability to access usual support [7, 9-11, 19]. A national survey has been performed in

Page 9/13 
the United Kingdom to prioritize mental health issues during the COVID-19 pandemic. Several concerns were reported, including mental illness, financial difficulties, but also family breakdown and increased domestic violence. Interestingly, consistent with our results, respondents were relatively less worried about becoming physically ill [40].

Furthermore, our results showed no association between domestic violence and PTSS, a stressor-related psychiatric disorder occurring after experiencing or witnessing events involving physical injury, death, or other threats to physical integrity [41]. As PTSD is one of the most common longterm psychiatric disorders, the absence of correlation could be related to insufficient time for the effect to appear. Furthermore, even when the lockdown is over, the consequences would still be detected months or even years after the COVID-19 pandemic ends [42]. Moreover, as the economic situation is deteriorating, abusers would be more likely to exert their power and aggression in the aftermath of a crisis and significant financial setbacks [3], further related mental health outcomes, especially PTSD.

\section{Public health recommendations.}

Implementing early adequate surveillance programs is warranted to capture the burden of gender differences and domestic violence on mental health during this pandemic [43]. Enhanced surveillance would provide targeted support and help developing intervention strategies the most vulnerable groups. Our study is of primary importance as it is the first to describe and analyze mental health-associated factors, particularly gender differences and domestic violence, in the context of "substantial gaps" regarding the surveillance and evaluation of effective interventions to support those at risk during this pandemic [44]. Hence, this contextual human rights analysis that took into account gender and violence as social and economic determinants of health. Considering such factors in future studies is paramount when developing interventions aiming at managing mental health problems.

\section{Limitations and strengths.}

Our study has some limitations. Since the data were collected online, the majority of participants were university graduates, with proper computer literacy and access to the Internet. Nevertheless, appropriate adjustments were made in the multivariate analysis taking into consideration several sociodemographic, economic, and COVID-19 related factors that can be potential confounders, including the level of education. Moreover, the sample size had an adequate power to assess correlations and potential confounding; nevertheless, residual confounding might still be possible. Another limitation could be related to the use of a self-reported questionnaire, possibly introducing an information bias, especially regarding the information related to violence in women, especially that we don't have any information related to whether couples were included in the study. However, the Arabic validated scales used had a very good to excellent reliability. This study can be considered a pilot one and it will be followed by other research better detailing violence in the Lebanese population. Despite these limitations, our study remains one of the few that performed a comparative and adjusted assessment of the combined effect of COVID-19 and a collapsing economy on mental health outcomes by gender and assess the effects of domestic violence. Additional studies taking into account all the weaknesses are suggested to confirm the current results.

\section{Conclusion}

Our study found that, in the context of combined health and economic crises, mental health outcomes are not equally felt by women or those subject to domestic violence. Women exhibit higher levels of stress, anxiety, and PTSS than men, while violent homes harbor more stress and insomnia. Additional studies in a few months would help better understand the long-term impact of the sanitary and economic crisis, especially on PTSS and mental well-being, which might not be perceptible during the crisis. Public health strategies are warranted to address, prevent, and manage transient and persisting psychiatric disorders, with focused measures to support and protect the most vulnerable populations, including women and those at high risk of domestic violence.

\section{Declarations}

Funding: The author(s) received no financial support for the research, authorship, and/or publication of this article.

Competing interests: The author(s) declared no potential conflicts of interest with respect to the research, authorship, and/or publication of this article.

Availability of data and materials: The datasets used and/or analyzed during the current study are available from the Figshare repository: https://figshare.com/s/939d0fc0032c7af0b8c9.

Ethics approval and consent to participate: The Institutional Review Board of the American University of Science and Technology approved this study protocol (AUST-IRB-20200527-01), as this work has been performed in accordance with the ethical standards laid down in the 1964 Declaration of Helsinki and its later amendments. The topic was explained to all participants in the introductory section of the survey and consent to participate was implicit. Anonymity of participants was guaranteed throughout the process of data collection and analysis.

Consent for publication: Not applicable.

Authors' contributions: All authors had full access to all data in the study and take responsibility for the integrity of the data and accuracy of analysis. $\mathrm{AH}$ and CAS contributed in literature search, study design, data collection, and drafting the manuscript. DB, HS, and RA contributed in literature search, 
data collection, and drafting the manuscript. PS designed the study, analysed the data, and contributed in data collection and drafting the manuscript.

Acknowledgements: The authors would like to thank all the persons who helped distribute the questionnaire, particularly Dr Fady Farhat, Dr Zahraa Shaitly, Mrs Joumana Sleilaty, and Mr Jihad Gerges.

\section{References}

1. Public Health Ministry and Information Ministry. Daily situation report 2020. [Available from: https://corona.ministryinfo.gov.lb, last accessed May 25, 2020.

2. Diwan I, Abi-Rached J, Lebanon. Managing Covid-19 in the Time of Revolution 2020 [Available from: https://www.arabreform.net/publication/managing-covid-19-in-the-time-of-revolution/.

3. Chbaro A. The Gendered Impact of COVID-19 in Lebanon. 2020 [Available from: https://daraj.com/en/42550/, Last accessed June 8, 2020.

4. Women Empowered for. Leadership, COVID-19: not the biggest threat for women in Lebanon. 2020.

5. Reuter. Lebanon closes schools until March 8 to curb coronavirus spread. 2020. [Available from: https://www.reuters.com/article/us-china-healthlebanon-education/lebanon-closes-schools-until-march-8-to-curb-coronavirus-spread-idUSKCN20M341, last accessed May 25, 2020.

6. Wenham C, Smith J, Morgan R, Gender, Group C-W. COVID-19: the gendered impacts of the outbreak. Lancet. 2020;395(10227):846-8.

7. Neil J. Domestic violence and COVID-19: Our hidden epidemic. Aust J Gen Pract. 2020;49.

8. Bradbury-Jones C, Isham L. The pandemic paradox: The consequences of COVID-19 on domestic violence. J Clin Nurs. 2020;29(13-14):2047-9.

9. Anurudran A, Yared L, Comrie C, Harrison K, Burke T. Domestic violence amid COVID-19. Int J Gynaecol Obstet. 2020;150(2):255-6.

10. van Gelder N, Peterman A, Potts A, O'Donnell M, Thompson K, Shah N, et al. COVID-19: Reducing the risk of infection might increase the risk of intimate partner violence. EClinicalMedicine. 2020:100348.

11. Chandan JS, Taylor J, Bradbury-Jones C, Nirantharakumar K, Kane E, Bandyopadhyay S. COVID-19: a public health approach to manage domestic violence is needed. Lancet Public Health 2020 [Internet]. 2020. Available from: https://www.thelancet.com/pdfs/journals/lanpub/PIIS24682667(20)30112-2.pdf. Last assessed: May 23, 2020.

12. Parkinson D. Investigating the Increase in Domestic Violence Post Disaster: An Australian Case Study. Journal of Interpersonal Violence. 2017;34(11):2333-62.

13. Taub A. A new COVID-19 crisis: Domestic abuse rises worldwide. The New York Times. 2020 [Available from: https://www.nytimes.com/2020/04/06/world/coronavirus-domestic-violence.html, Last accessed June 12, 2020.

14. The Guardian. Lockdowns around the world bring rise in domestic violence. 2020.

15. Boserup B, McKenney M, Elkbuli A. Alarming trends in US domestic violence during the COVID-19 pandemic. Am J Emerg Med. 2020.

16. Rahme C, Haddad C, Akel M, Khoury C, Obeid H, Obeid S, et al. Factors associated with violence against women in a representative sample of the Lebanese population: results of a cross-sectional study. Arch Womens Ment Health. 2020.

17. Usta J, Farver JA, Pashayan N. Domestic violence: the Lebanese experience. Public Health. 2007;121(3):208-19.

18. Awwad J, Ghazeeri G, Nassar AH, Bazi T, Fakih A, Fares F, et al. Intimate Partner Violence in a Lebanese Population Attending Gynecologic Care: A Cultural Perspective. J Interpers Violence. 2014;29(14):2592-609.

19. World Health Organaization (WHO). Joint Leaders' statement - Violence against children: A hidden crisis of the COVID-19 pandemic 2020 [Available from: https://www.who.int/news-room/detail/08-04-2020-joint-leader-s-statement--violence-against-children-a-hidden-crisis-of-thecovid-19-pandemic, last accessed May 22, 2020.

20. Casagrande M, Favieri F, Tambelli R, Forte G. The enemy who sealed the world: Effects quarantine due to the COVID-19 on sleep quality, anxiety, and psychological distress in the Italian population. Sleep Medicine. 2020.

21. Ozdin S, Bayrak Ozdin S. Levels and predictors of anxiety, depression and health anxiety during COVID-19 pandemic in Turkish society: The importance of gender. Int J Soc Psychiatry. 2020:20764020927051.

22. Liu N, Zhang F, Wei C, Jia Y, Shang Z, Sun L, et al. Prevalence and predictors of PTSS during COVID-19 outbreak in China hardest-hit areas: Gender differences matter. Psychiatry Res. 2020;287:112921.

23. Ahorsu DK, Lin CY, Imani V, Saffari M, Griffiths MD, Pakpour AH. The Fear of COVID-19 Scale: Development and Initial Validation. Int J Ment Health Addict. 2020:1-9.

24. Barbour B, Saadeh N, Salameh P. Psychological distress in Lebanese young adults: constructing the screening tool 'BDS-22.'. International Journal of Culture Mental Health. 2012;5(2):94-108.

25. Hallit S, Obeid S, Haddad C, Hallit R, Akel M, Haddad G, et al. Construction of the Lebanese Anxiety Scale (LAS-10): a new scale to assess anxiety in adult patients. Int J Psychiatry Clin Pract. 2020:1-8.

26. Ibrahim H, Ertl V, Catani C, Ismail AA, Neuner F. The validity of Posttraumatic Stress Disorder Checklist for DSM-5 (PCL-5) as screening instrument with Kurdish and Arab displaced populations living in the Kurdistan region of Iraq. BMC Psychiatry. 2018;18(1):259. 
27. Hallit S, Sacre H, Haddad C, Malaeb D, Al Karaki G, Kheir N, et al. Development of the Lebanese insomnia scale (LIS-18): a new scale to assess insomnia in adult patients. BMC Psychiatry. 2019;19(1):421.

28. Good M-J, Smilkstein G, Good B, Shaffer T, Arons T. The Family APGAR Index: A Study of Construct Validity. The Journal of Family Practice. 1979;8(3):577-82.

29. Sibai AM, Chaaya M, Tohme RA, Mahfoud Z, Al-Amin H. Validation of the Arabic version of the 5-item WHO Well Being Index in elderly population. Int J Geriatr Psychiatry. 2009;24(1):106-7.

30. Prawitz A, Garman ET, Sorhaindo B, O’Neill B, Kim J, Drentea P. Incharge Financial Distress/Financial Well-Being Scale: Development, Administration, and Score Interpretation Financial. Counseling Planning. 2006;17(1):34-50.

31. Huang Y, Zhao N. Generalized anxiety disorder, depressive symptoms and sleep quality during COVID-19 outbreak in China: a web-based crosssectional survey. Psychiatry Res. 2020;288:112954.

32. Mishra P, Pandey CM, Singh U, Gupta A, Sahu C, Keshri A. Descriptive statistics and normality tests for statistical data. Ann Card Anaesth. 2019;22(1):67-72.

33. Altemus M, Sarvaiya N, Neill Epperson C. Sex differences in anxiety and depression clinical perspectives. Front Neuroendocrinol. 2014;35(3):32030.

34. Wang C, Zhao H. The Impact of COVID-19 on Anxiety in Chinese University Students. Front Psychol. 2020;11:1168.

35. Moghanibashi-Mansourieh A. Assessing the anxiety level of Iranian general population during COVID-19 outbreak. Asian J Psychiatr. 2020;51:102076.

36. Felmingham K, Williams LM, Kemp AH, Liddell B, Falconer E, Peduto A, et al. Neural responses to masked fear faces: sex differences and trauma exposure in posttraumatic stress disorder. J Abnorm Psychol. 2010;119(1):241-7.

37. Francis L, Loxton D, James C. The culture of pretence: a hidden barrier to recognising, disclosing and ending domestic violence. J Clin Nurs. 2017;26(15-16):2202-14.

38. Gallegos AM, Trabold N, Cerulli C, Pigeon WR. Sleep and Interpersonal Violence: A Systematic Review. Trauma Violence Abuse. 2019:1524838019852633.

39. Ophuis RH, Olij BF, Polinder S, Haagsma JA. Prevalence of post-traumatic stress disorder, acute stress disorder and depression following violence related injury treated at the emergency department: a systematic review. BMC Psychiatry. 2018;18(1):311.

40. The Academy of Medical Sciences. Survey results: Understanding people's concerns about the mental health impacts of the COVID-19 pandemic 2020 [Available from: https://acmedsci.ac.uk/file-download/99436893.

41. American Psychiatric Association. Diagnostic and statistical manual of mental disorders. 5 ed. Washington: DC; 2013.

42. Brooks SK, Webster RK, Smith LE, Woodland L, Wessely S, Greenberg N, et al. The psychological impact of quarantine and how to reduce it: rapid review of the evidence. Lancet. 2020;395(10227):912-20.

43. Galea S, Merchant RM, Lurie N. The Mental Health Consequences of COVID-19 and Physical Distancing: The Need for Prevention and Early Intervention. JAMA Intern Med. 2020.

44. Chandan JS, Taylor J, Bradbury-Jones C, Nirantharakumar K, Kane E, Bandyopadhyay S. COVID-19: a public health approach to manage domestic violence is needed. Lancet Public Health. 2020;5(6):e309.

\section{Figures}




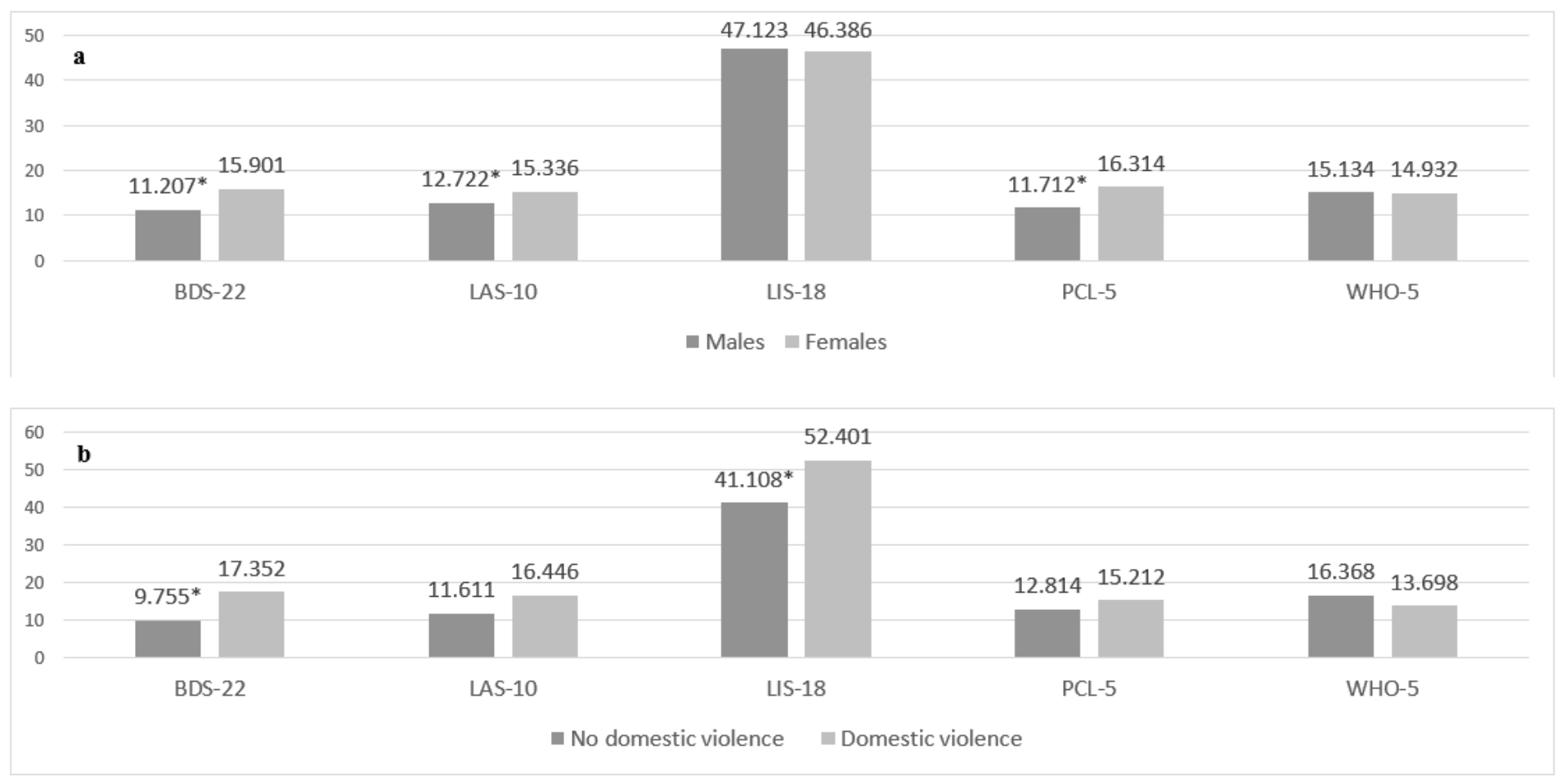

\section{Figure 1}

Mental Health Measure: Estimated marginal means per gender (a) and according to domestic violence (b). ${ }^{*} P<0.05$. a) $P$-values for BDS-22=0.005; LAS-10=0.033; LIS-18=0.645; PCL-5=0.025 and WHO-5=0.754. Adjustment over: age, marital status, university education, health coverage, chronic disease, family member with chronic disease, worry for family member with chronic disease, fear of no access to chronic disease treatment, domestic violence, professional status, socioeconomic status, APGAR family scale, financial wellness and fear of COVID. b) p-values for BDS-22=0.041; LAS$10=0.078$; LIS-18=0.002; PLC-5=0.601 and WHO-5=0.065. Adjustment over: gender, age, marital status, university education, health coverage, chronic disease, family member with chronic disease, worry for family member with chronic disease, fear of no access to chronic disease treatment, professional status, socioeconomic status, APGAR family scale, financial wellness and fear of COVID. 